\title{
Nivelación propuesta por la SENESCYT: vivencias en la universidad central del Ecuador
}

\section{Leveling proposed by the SENESCYT: experiences at the central university of Ecuador}

Luis Buitrón

Jorge Ortiz

Yolanda Yupangui

Universidad Central del Ecuador, Ecuador

Autores para correspondencia: hbuitron@uce.edu.ec, jdortiz@uce.edu.ec, ymyupangui@uce.edu.ec

Fecha de recepción: 20 de Agosto de 2017 - Fecha de aceptación: 30 de Septiembre de 2017

Resumen: En la educación superior existe un problema crítico y permanente, el cual corresponde a la admisión en los estudios superiores de los bachilleres formados por la educación media. La Universidad Central del Ecuador ha formulado diferentes respuestas en las últimas décadas, sin embargo la SENESCYT ha impuesto un modelo creado desde la discusión teórica y éste ha sido obligatorio desde 2013. La investigación buscó verificar su aporte a la solución del problema y comparar con la admisión inmediata anterior y una propuesta planteada en 2016, la verificación se realiza en tres Facultades de las dieciocho existentes; para aquello se analizó el rendimiento en matemática y lenguaje, así como la adaptación en el nivel de educación superior. Los resultados evidencian que no se pueden generalizar entre las Facultades estudiadas, la propuesta de SENESCYT provoca mejoras en Carreras con libre ingreso, pero no en los otros casos y se descubre que es necesario una propuesta nueva.

Palabras claves: nivelación de carrera; currículo; sistema nacional de nivelación y admisión; aspirantes

Abstract: In the superior education a critical and permanent problem exists, which corresponds to the admission in the superior studies of the high schools formed by the half education. The Central University of the Ecuador has formulated different answers in the last decades, however the SENESCYT has imposed a model created from the theoretical discussion and this has been obligatory from 2013. The investigation looked for to verify its contribution to the solution of the problem and to compare with the previous immediate admission and a proposal outlined in 2016, the verification is carried out in existent three Faculties of eighteen; for that the yield was analyzed in mathematics and language, as well as the adaptation in the level of superior education. The results evidence that they cannot be generalized among the studied Faculties, the proposal of SENESCYT causes improvements in Careers with free entrance, but not in the other cases and is discovered that it is necessary a new proposal.

Key words: leveling of career; curriculum; national system of leveling and admission; applicant 


\section{Introducción}

El nivel de bachillerato en el Ecuador ha tenido una progresión, se parte desde la propuesta de1978, ahí se especificaron dos tipos de bachilleratos, el primero en ciencias con las especialidades de: físico-matemáticas, química-biológicas, ciencias sociales y combinaciones de éstos; el segundo el bachillerato técnico con cerca de treinta especialidades, agrupadas en las técnico-industriales, técnicas-comerciales, agrícolas y en artes, esto como resultados del Plan Integral de Transformación y Desarrollo 1973-1977, promovido desde el Gobierno, cuya política se orientó a promover el conocimiento de la realidad nacional y pretendió reflejar el espíritu transformador de los dictadores encargados del Poder (Ministerio de Educación y Cultura, 1994).

Con algunas reformas el modelo de bachillerato permanece en su estructura hasta marzo de 2011, en ese tiempo se permitió iniciativas desde la Instituciones de Nivel Medio, entre ellas, los colegios experimentales, lo que permitió realizar algunas mejoras en las propuestas curriculares que aportaron en la mejora en la formación del Bachiller y el nivel de educación superior asumió, en cierta medida, esos perfiles para la admisión de estudiantes en las diferentes Carreras; el fin de este modelo termina cuando se emite la Ley Orgánica de Educación Intercultural (LOEI) y debe construirse el nuevo modelo (Asamblea Nacional, 2012).

A partir de la LOEI, el Ministerio de Educación implementó el Nuevo Bachillerato Ecuatoriano, que genera una propuesta única de formación, los primeros graduados del nivel se tienen en el 2014. El llamado Bachillerato General Unificado (BGU) (Ministerio de Educación, 2010) agrupa a los bachilleratos en ciencias en su totalidad con cuarenta horas de clase semanales para el desarrollo del llamado "Tronco Común" y a los técnicos con 45 horas semanales que estudiarán a más del tronco común el componente de la formación técnica en un promedio de 15 horas a la semana (10, 10 y 25 horas por semana en cada año de bachillerato).

Para el ingreso a la Universidad con las regulaciones de la Secretaría de Educación Superior, Ciencia, Tecnología e Innovación (SENESCYT, 2012), en un primer momento se presentaron los bachilleratos con especialidades y desde 2014 participan los de bachillerato unificado. El cambio en el perfil de salida del bachiller no modificó el currículo del curso de nivelación que plantea y exige la Secretaría, por su parte las Universidades tampoco han adaptado sus acciones a la presencia de un nuevo bachiller.

Una de las aspiraciones de las Universidades, en varios casos errores comunes, es pretender encontrar estudiantes con cualidades superiores en el universo de aspirantes, que ellos tengan capacidades de alto (o muy alto) razonamiento en lo numérico, lógico y verbal; aquello traslada a situaciones irreales en el punto de partida en la formación de Carrera y hace necesaria a la nivelación de Carrera. Surgen varias preguntas ¿Qué debe incluir la nivelación?, ¿Cuál es el punto de partida de la nivelación?, ¿Hasta dónde debe alcanzar el trabajo de nivelación?, ¿La propuesta curricular única por área de la SENESCYT es adecuada, viable y sostenible?, ¿Qué docencia debe conducir la nivelación?

En la Universidad Central del Ecuador (UCE) existieron diversos criterios de admisión, desde el libre ingreso heredado de la década de los setenta, hasta la aplicación de exámenes de conocimientos y psicométricos, en el caso de la Facultad de Ingeniería en Geología, Minas, Petróleos y Ambiental (FIGEMPA) los doce años previos al "examen nacional para el ingreso a 
la educación superior" ENES, se aplicaron las dos tipos de pruebas, las académicas y las psicométricas, sus resultados reflejaron alta correlación entre sí, en los primeros ocho años se promocionaban a Carrera los mejores puntajes según el cupo previsto (120 a 200 por período), en lo posterior (por dos años), quienes superaban el puntaje equivalente al $70 \%$ se promocionaban a primer semestre, lo que determinó hasta quince estudiantes por semestre, que equivalía a menos del 3\% de los inscritos (Ortiz, 2010) y los siguientes estudiantes según el cupo a nivelación en las tres asignaturas requeridas (matemática, física y química); en los dos años previos a la política pública emitida desde el Gobierno, el acceso a primer semestre se asignó por puntaje y en función de los cupos determinados por Carrera (entre veinte y cuarenta). En el caso de Derecho, Sociología, Política y Trabajo Social, Facultad de Jurisprudencia, la admisión se realizó bajo el criterio de libre ingreso, lo que significó que los estudiantes que se inscribieron obtuvieron su cupo y cursaron cada Carrera según el caso. En el caso de la FAU la admisión se realizó por medio del curso de nivelación a los estudiantes inscritos y la aplicación de pruebas, de ahí se seleccionó a los estudiantes que pasaron a Carrera.

Es por ello que la universidad ecuatoriana debe estudiar esta realidad en función de explicar lo que ha sucedido en el ingreso a la educación superior y sobre todo ajustar acciones y desarrollar programas conducentes a que la transición de la educación media y superior sea un evento natural en el itinerario educativo de sus estudiantes.

Se propuso en la investigación el determinar el nivel de cumplimiento de los objetivos del Sistema Nacional de Nivelación y Admisión (SNNA) en las Facultades de Arquitectura y Urbanismo (FAU); Ingeniería en Geología, Minas, Petróleos y Ambiental (FIGEMPA); y, Jurisprudencia, Ciencias Políticas y Sociales (FJCPS) de la UCE.

Con sus resultados se generarán elementos de reflexión académica, evaluar los resultados obtenidos en las tres Facultades señaladas, finalmente orientarán a futuros procesos de ingreso de estudiantes.

\section{Marco teórico}

\section{Admisión Universitaria}

La admisión en el sistema de educación superior ha experimentado cambios en América Latina a lo largo de la última década; con respecto al aumento de estudiantes en las Instituciones de Educación Superior (IES), (Yamada, Castro, Bacigalupo, \& Velarde, 2013) manifiestan que:

Si bien un incremento en el acceso a la educación superior es deseable como herramienta para el desarrollo económico y humano del país, es necesario que venga acompañado de reglas de juego que preserven sistemas de admisión relativamente exigentes. Asimismo, la educación básica debe incrementar sustantivamente los aprendizajes de los estudiantes. De nada sirve tener un mayor porcentaje de nuestros jóvenes con educación superior completa si al llegar al mercado laboral lo único que obtendremos será una mayor proporción de desempleados con títulos. (p. 29)

Aunque también es posible incrementar el número de estudiantes en educación superior sin disminuir la calidad académica, como es el caso de Corea del Sur, que ha alcanzado la 
cobertura total en este nivel escolar, y que lo ha hecho manteniendo altos estándares de calidad; así como Chile, con una de las más altas tasas de cobertura en América Latina" (Ocegueda, Miramontes, Moctezuma, \& Mungaray, 2017) (p. 142).

En este sentido, les debe corresponder a las Instituciones de Educación Superior (IES), garantizar que el currículo, de nivelación y de formación profesional, responda a los requerimientos académicos del claustro, a las necesidades del mercado laboral y sobre todo a los requerimientos de la Sociedad requirente de titulados probos, para que la educación superior en este contexto sea de excelente calidad.

Desde la perspectiva más general, el proceso de democratizar la educación superior y el conocimiento el cual según (Ramírez, 2013) "está basado fundamentalmente en igualar las competencias/destrezas (no conocimientos)", debe cumplirse de manera que una vez que el estudiante admitido haya aprobado el curso de nivelación, esté apto para insertarse en el mundo universitario con éxito.

En el Ecuador, han pasado algo más de cuarenta años de la eliminación formal del examen de ingreso, por ello operan variados mecanismos establecidos por las mismas instituciones educativas, aquello impide conocer el estado de las políticas de admisión y nivelación a la educación superior (Senplades, 2010), y no permite la construcción de una política pública, por ello desde la Secretaría Nacional de Planificación y Desarrollo se motivó escenarios de discusión y propuesta, esto influye en los preceptos constitucionales y es, con mucha seguridad, el precedente orientador a la propuesta de acceso a la educación superior propuesta desde la SENESCYT.

El acceso de estudiantes a la educación superior pública se lo realiza a través de Sistema Nacional de Nivelación y Admisión en el marco de gratuidad, establecida en la Constitución de 2008. El sistema tiene tres componentes: 1) la aplicación del "examen nacional de educación superior" y la obtención del puntaje de cada aspirante, aquello le permite postular a determinada Carrera y esperar la asignación de cupo, 2) La asignación a la Carrera y con ello la posibilidad de realizar la nivelación o en caso de presentarse al examen "exonera" promoverse a primer nivel, y 3) aprobación de nivelación y promoción a primer nivel de Carrera (SENESCYT, 2014).

A partir de la Constitución de 2008, se define al buen vivir como una nueva forma de convivencia ciudadana, en diversidad y armonía con la naturaleza tal como señala la Constitución, además en su artículo 26 se hace referencia al derecho que tienen las personas a la educación ya que es un deber ineludible e inexcusable del Estado, donde se garantiza la igualdad e inclusión social como condición indispensable para el buen vivir (Asamblea Constituyente, 2008). Asimismo, en el artículo 10 de la Ley Orgánica de Educación Superior (LOES), se hace referencia a la articulación del sistema educativo: "La educación superior integra el proceso permanente de educación a lo largo de la vida. El Sistema de Educación Superior se articulará con la formación inicial, básica, bachillerato y la educación no formal" (Asamblea Nacional, 2010).

En este sentido, (Ramírez, 2013) manifiesta que "No sería apresurado si señalásemos que el corazón para producir un acompañamiento de la universidad en la edificación de la sociedad 
del Buen Vivir reside en el cambio radical del régimen académico", para lograr una articulación adecuada entre los diferentes niveles del sistema educativo, hay que determinar políticas públicas que permitan al estudiante insertarse en la vida universitaria.

El módulo de buen vivir, propuesto por el SNNA, tiene como unidad de análisis central el desarrollo de las habilidades básicas del pensamiento, estableciendo una experiencia educativa, cuya aplicación y experimentación basada en la reflexividad, genere la producción del proyecto de vida y destrezas en la organización de los aprendizajes de la educación superior.

El proyecto de vida, que es una unidad destinada hacia la reflexividad de los aspirantes basada en las preguntas fundacionales ¿Quién soy?, ¿de dónde vengo?, ¿Hacia dónde voy? y ¿Cómo lo voy a hacer?; proyecto de vida que permitirá al aspirante planificar su vida futura en las diversas dimensiones personales, familiares, universitarias, culturales y profesionales (Barreto, Lescano, Hidalgo, Inca, \& Cisneros, 2017).

Es visible que la propuesta de "Universidad y el buen vivir" tiene un peso gravitante, por tanto hay que esperar que aporte de manera significativa al estudiante durante su nivelación, la investigación pretende validar todo el interés dado desde la Secretaría de Estado.

\section{Nivelación de Carrera por SNNA}

La SENESCYT propone un currículo para nivelación que se organiza en seis áreas de conocimiento: 1) Ciencias, Ingenierías, Industria y Construcción; 2) Agricultura; 3) Artes; 4) Programas Básicos, Educación, Servicios, Ciencias Sociales y Derecho; 5) Educación Comercial, Economía y Afines; y 6) Salud (SENESCYT, 2014). Para la UCE, las Carreras de: Arquitectura e Ingenierías en: Geología, Minas, Petróleos y Ambiental, se agrupan en el área 1; en el caso de las carreras de Derecho, Sociología, Política y Trabajo Social, se agrupan en el área 4. La primera área citada dedica 140 horas al trabajo de las asignaturas de tronco común: Introducción a la comunicación científica (60h), Universidad y buen vivir (60h) y Proyecto integrador de saberes (20h); el tiempo restante, 400 horas, se dedica a las asignaturas de Matemáticas (200h), Física (100h), y Química (100h). La segunda área a más del tronco común asigna las 400h horas restantes a las asignaturas de: Matemática (140h), Sociedad y Cultura (140h) y Psicología (120h). En la UCE en un primer momento se cumplió con las horas señaladas, pero luego de realizar evaluaciones internas en 2015, el tronco común se ejecutó al asignar siete de las treinta horas de trabajo semanal, las veinte y tres horas restantes para las asignaturas específicas.

\section{Propuesta de nivelación UCE}

Dentro de la reflexión a partir de factores internos y externos la UCE ha considerado elementos que le permitan realizar una propuesta que responda mejor a sus requerimientos, es así que participamos de la clasificación afirmada por Paola Viera en 2017 al definir a "los que triunfan y los que fracasan" (Viera, 2017), su referente sobre el proceso de 2014, en el que hay estudiantes que reprueban el ENES (36.054 estudiantes) al no superar el puntaje mínimo de 600/1000 y los sin cupo (165.627) que corresponden al $80 \%$ de los aplicantes, ellos son los que fracasan, por el lado de los que triunfan hay los con cupo (49.049) y los del grupo de alto rendimiento (GAR) (264) y equivalen al $20 \%$. 
Así como lo afirma Betzabé Maldonado, la nivelación debería cumplir los objetivos de: 1) establecer mecanismos de equiparación de conocimientos del bachillerato, 2) abordar la preparación para el inicio de la carrera y orientación académica, y ratifica que los resultados son poco favorables para la inserción de los estudiantes a las carreras universitarias (Maldonado, 2015), en los resultados de la UCE éstos deberán ser verificados para reorientar el proceso, sin embargo se remite tasas de repetición significativas, en varios casos superando índices del 40\%, de manera especial en las ingenierías.

Cecilia Carrera al realizar una investigación sobre el proceso de admisión en la Escuela Politécnica Nacional, se refiere en una de sus conclusiones a que se debe mejorar el proceso de selección de docentes de nivelación y debe incluirse la capacitación pedagógica, aptitudes para la docencia y experiencia en cátedra (Carrera, 2017), y se enfatiza que hay una percepción en la que predomina la empleabilidad por sobre la responsabilidad consiente de realizar la nivelación previo al ingreso, la certificación de la SENESCYT a los docentes habilitados se basó en la aprobación de evaluaciones y aquello no es suficiente para reconocer la empatía con la responsabilidad profesional requirente.

Al analizar, en la Dirección General Académica (DGA), las 140 h correspondientes al tronco común se concluye que son espacios extendidos frente a lo que aporta dentro de la nivelación según lo esperado por las Carreras y que desde las Facultades hay una presión para una verdadera nivelación de conocimientos, muchos de ellos no adquiridos en las etapas de formación anteriores, es ahí que desde la DGA se propone una formulación diferente de organización educativa y curricular.

Un factor imponderable para la Secretaría fue el presupuestario, para el proceso 20162017 no contó con recursos y solo esa medida le lleva a declinar su postura frente al cumplimiento del currículo y horas de trabajo por cada área, en ese momento se viabiliza la propuesta de la UCE, que fue negada por la Gerencia de SNNA un año anterior.

La propuesta que organiza a las Carreras en ocho áreas: 1) Educación; 2) Humanidades y Artes; 3) Administración y Economía; 4) Ciencias de la Vida; 5) Ingeniería, Industria y Construcción; 6) Agricultura; 7) Salud y Servicios Sociales; y 8) Ciencias Sociales y Derecho. Cada área tendrá cuatro asignaturas una común que corresponde a "Ética y Pensamiento Universitario" y tres particulares para cada área.

La asignatura común se desarrolla en plataforma virtual, lo cual da un valor agregado desde el currículo, además de ser flexible para su implementación, esta asignatura persigue la inducción estudiantil y el asumir la responsabilidad dentro del mundo del conocimiento. Las tres asignaturas de cada área plantean priorizar una de ellas y complementar con otras dos, la carga horaria propuesta es ocho horas semanales para la primera y seis para las complementarias. El tiempo ideal marcado es por un período de 16 semanas, en el primer semestre por la restricción económica se reduce a diez semanas.

\section{Marco Metodológico}

La investigación se realizó con enfoque cuantitativo, lo que permitió buscar información por medio de encuestas y entrevistas; también se realizó búsqueda de información en las bases de 
datos de la UCE, luego de su análisis se obtienen los resultados con respecto al cumplimiento de objetivos del proyecto curricular de nivelación propuesto por el SNNA-SENESCYT.

El estudio tiene, es también exploratorio puesto que por primera vez se realiza una investigación sobre nivelación en la UCE en el período 2012-2016, los datos del período 2012 referencia a la práctica anterior a la propuesta, los de 2014 reflejan la propuesta de la Secretaría y los datos de los estudiantes que cursaron en 2016 proveen de información de la propuesta de la Universidad.

Otras características del trabajo se enmarcan en se descriptico, transversal, retrospectivo y comparativo.

En la recolección de información se consideró a los estudiantes que hicieron nivelación en los semestres 2012-2012, 2014-2014, 2016-2016; a los docentes (a la fecha: 2017) de Lenguaje y Matemática de primer semestre de las carreras de las facultades de Arquitectura, FIGEMPA Y Jurisprudencia de la UCE. Se consideraron los siguientes criterios de exclusión: Estudiantes que rindieron y aprobaron examen exonera, estudiantes que cursaron el primer nivel de una carrera y aplican al primer nivel de otra carrera de la misma u otra universidad pública, los estudiantes que corresponden al $10 \%$ de población que se cambia de una universidad privada y aquellos que perdieron la asignatura por haberse retirado por varias causas.

La Dirección de Tecnologías, Información y Comunicación (DTIC) de la UCE proporcionó la base de datos de los estudiantes que en los años 2012, 2014 y 2016 cursaron nivelación (población universo), de esta manera se procesó la información por Facultad, por Carrera y por periodo. Con ello se determinó la información de los estudiantes con respecto a las destrezas, habilidades y características desarrolladas como por ejemplo, razonamiento numérico, lógico y verbal, así como su inmersión en el mundo universitario.

A pesar de que el tema de investigación involucra a personas, no se trató de ensayar en seres humanos para conocer la eficiencia de un sistema académico aplicado, por el contrario se buscaron los aciertos y las deficiencias que lleven a uno u otro resultado, lo que permitirá sugerir correctivos de ser necesario, lo cual es beneficioso para las futuras generaciones de estudiantes que aspiren a la educación superior de la UCE.

\section{Descriptores}

Para determinar el nivel de cumplimiento de los objetivos del SNNA en las Facultades señaladas se operacionaliza el objetivo en:

1) Determinar a través del análisis comparativo entre los porcentajes de repitencia de los estudiantes de primer semestre en diferentes períodos.

2) Verificar la utilidad del curso de nivelación en cuanto permite a los nuevos estudiantes superar el inicio de la carrera, sin dificultades en operaciones de razonamiento lógico, numérico y verbal.

3) Desarrollar las habilidades de comunicación oral y escrita, en el marco del desarrollo de sus capacidades para el manejo de los diversos lenguajes de la ciencia, la profesión y las humanidades. 
4) Establecer si se aplicaron los principios de interculturalidad y respeto a la diversidad en el trabajo de equipos colaborativos de estudio.

\section{Resultados}

Sobre repetición de las asignaturas de matemática y lenguaje, obtenidos del Sistema Académico Universitario (SAU), los resultados son diversos, no es posible determinar a partir de ellos la influencia en la repetición de las asignaturas descritas por el mecanismo de nivelación cursado por los estudiantes. Se ha reconocido que hay dos factores que gravitaron de manera significativa, el primero es que la docencia tuvo cambios en este período, en esos años hubo cambio en casi la totalidad de los docentes que fueron responsables de éstas asignaturas y los que hoy son; el segundo cambio tiene que ver con las modificaciones curriculares planificadas, en un inicio había un enfoque a la planificación por competencia (como resultado de las políticas curriculares de la década anterior) y hoy se orienta a los resultados de aprendizaje (como influencia gravitante de las disposiciones legales).

En referencia a la utilidad del curso de nivelación y que el estudiante pueda superar el inicio de la carrera y que no presenten dificultades en operaciones de razonamiento lógico, numérico y verbal se han obtenido los siguientes resultados:

1. En el caso de la FJCPS en el desarrollo de razonamiento en lo referente al desarrollo de pensamiento en 2012 se logró un 56\% de respuestas afirmativas, en los años posteriores se mantuvo (59\% y 55\%). En el desarrollo de habilidades de comunicación oral parte de un $68 \%$, reduce a $48 \%$ y termina en $71 \%$, lo cual hace notar que la propuesta de Senescyt fue regresiva. En las habilidades de comunicación escrita los porcentajes son $52 \%, 58 \%$ y $64 \%$; en cuyo caso afirmaremos que hay una evolución.

2. En la FAU en el desarrollo de pensamiento, en 2012, da un 50\% de respuestas afirmativas, en los años posteriores se mantuvo (63\% y 60\%). En el desarrollo de habilidades de comunicación oral parte de un $75 \%$, reduce a $63 \%$ y termina en $43 \%$, lo cual hace notar que la propuesta ha influenciado de manera negativa. En las habilidades de comunicación escrita los porcentajes son $71 \%$, 60\% y 58\%; también hay un retroceso significativo.

3. En FIGEMPA en el desarrollo de pensamiento, parte de un $86 \%$, se reduce a $67 \%$ y regresa a $86 \%$. En el desarrollo de habilidades de comunicación oral parte de un $57 \%$, reduce a $54 \%$ y se recupera a 79\%, al final hay una mejora. En las habilidades de comunicación escrita los porcentajes son $86 \%, 65 \%$ y $71 \%$; es necesario dar énfasis.

4. Los niveles de satisfacción en el desarrollo con el razonamiento lógico en la FJCPS parten del 52\% y varían al 38\% y 49\% en los siguientes cohortes, la variación resultó negativa y casi recupera el valor inicial; en la satisfacción en el desarrollo del razonamiento numérico varía del 28\%, 38\% y 43\% habiendo una progresión; finalmente, en el razonamiento verbal los resultados son $56 \%, 58 \%$ y $61 \%$; habiendo un progreso leve en cada período.

5. En la FAU, los niveles de satisfacción en el desarrollo con el razonamiento lógico parten del $54 \%$ y varían al $60 \%$ y $70 \%$, la variación presenta mejoras; en la satisfacción en el desarrollo del razonamiento numérico varía del 50\%, 87\% y 85\% 
logrando resultados satisfactorios; en el razonamiento verbal los resultados son $50 \%$, $43 \%$ y $35 \%$; habiendo un progreso negativo en cada período.

6. En la FIGEMPA, los niveles de satisfacción en el desarrollo con el razonamiento lógico son $57 \%, 67 \%$ y $57 \%$ respectivamente, la propuesta de Senescyt muestra un valor superior a los otros; en la satisfacción en el desarrollo del razonamiento numérico varía del $71 \%, 67 \%$ y $64 \%$ habiendo un descenso leve en cada caso; finalmente, en el razonamiento verbal los resultados son 57\%, 46\% y 36\%; habiendo deterioro significativo en cada período.

Uno de los tres objetivos del bachillerato es prepararlo para cursar con éxito la educación superior, por tanto se tomaron elementos del perfil de salida del bachiller y se investigó sobre la satisfacción en el avance de estas capacidades, lo cual provee los siguientes resultados:

7. En la FJCPS, la capacidad de pensar en modo crítico tuvo como resultados a 44\%, $44 \%$, y $44 \%$ en los tres períodos, mostrando una constante; en la capacidad de comunicarse efectivamente los valores son de $44 \%, 44 \%$ y $53 \%$, en el tercer período se presenta una mejora, en la capacidad de razonar numéricamente los resultados son $24 \%, 33 \%$ y $43 \%$; mostrando mejora; en la capacidad de utilizar herramientas tecnológicas de forma reflexiva y práctica los resultados son $40 \%, 48 \%$ y $34 \%$; en la capacidad de comprender su historia, realidad social y natural se tiene $44 \%, 59 \%$ y $56 \%$ reconociendo una mejora con respecto al primer cohorte.

8. En la FAU, la capacidad de pensar en modo crítico tuvo como resultados a $33 \%, 50 \%$, y $58 \%$ en los tres períodos, mostrando una mejora constante; en la capacidad de comunicarse efectivamente los valores son de $42 \%, 40 \%$ y $58 \%$, en el tercer período se presenta una mejora, en la capacidad de razonar numéricamente los resultados son $50 \%, 80 \%$ y $75 \%$; mostrando mejora; en la capacidad de utilizar herramientas tecnológicas de forma reflexiva y práctica los resultados son $25 \%, 40 \%$ y $50 \%$, resulta una mejora continua; en la capacidad de comprender su historia, realidad social y natural se tiene $38 \%, 27 \%$ y $48 \%$ en esta capacidad la propuesta de la SENESCYT es un retroceso.

9. En la FIGEMPA, la capacidad de pensar en modo crítico tuvo como resultados a $43 \%, 48 \%$, y $57 \%$ en los tres períodos, mostrando mejoras; en la capacidad de comunicarse efectivamente los valores son de $42 \%, 40 \%$ y $58 \%$, en el tercer período se presenta mejora, en la capacidad de razonar numéricamente los resultados son $50 \%, 80 \%$ y $75 \%$; los dos períodos muestran mejoras considerables respecto del 2012; en la capacidad de utilizar herramientas tecnológicas de forma reflexiva y práctica los resultados son $57 \%, 48 \%$ y $50 \%$; en la capacidad de comprender su historia, realidad social y natural se tiene $14 \%, 42 \%$ y $43 \%$ reconociendo una considerable mejora con respecto al primer cohorte.

Se logró describir adaptación en la Universidad; la organización, normativa, valores, principios y gestión universitaria y la adquisición de dominios de instrumentos básicos para desenvolvimiento en universidad en los siguientes términos:

1. En la inmersión al mundo universitario los estudiantes de la FJCPS de un valor de $60 \%$ hay reducciones leves al 58\% y 53\%. En el caso de la FAU parte del 54\%, sube 
al $57 \%$ y al $75 \%$, la mejora es considerable. Para la FIGEMPA se parte del $71 \%$, se reduce al $63 \%$ y se regresa al $71 \%$.

2. Durante la nivelación el factor que logra más identificación en los estudiantes en el trabajo en los "Principios y valores universitarios", con un valor del 47\% (FJCPS $51 \%$, FAU 43\%, FIGEMPA 46\%), en segundo lugar está la "organización universitaria" con 22\% (FJCPS 22\%, FAU 18\%, FIGEMPA 26\%), en tercer lugar y cercano al anterior se encuentra la "Normativa" con 19\% (FJCPS 17\%, FAU 24\%, FIGEMPA 16\%) y finalmente el último elemento que se reconoce como factor identificado es la "Gestión universitaria" (FJCPS 10\%, FAU 15\%, FIGEMPA 12\%).

En lo referente a los principios de interculturalidad y al respeto a la diversidad (trabajo en equipos colaborativos), se tienen los siguientes resultados:

1. En lo referente a la identificación con los principios de interculturalidad la FJCPS parte de un valor de $44 \%$, hay mejoras a $53 \%$ y $69 \%$ lo cual es satisfactorio. En el caso de la FAU parte del 63\%, baja al $43 \%$ y mejora al $88 \%$, el impacto de la propuesta de la SENESCYT es negativo, se recupera de manera significativa. Para la FIGEMPA se parte del $86 \%$, se reduce al $65 \%$ y se regresa al $86 \%$.

2. Los resultados muestran una alta sintonía entre lo realizado en nivelación y los principios identificados en la interculturalidad como medio de convivencia socioafectiva, en general no existen rechazos en los niveles de satisfacción al dominar el nivel de satisfacción, poca satisfacción y muy satisfactorio, quedan en los últimos lugares lo insatisfactorio y muy insatisfactorio.

\section{Conclusiones}

El curso de Nivelación de carrera propuesto por la SENESCYT ha cumplido con los objetivos planteados en su propuesta curricular.

El perfil de egreso de los estudiantes de nivelación, se articula con las asignaturas antecesoras para que los estudiantes asuman los contenidos curriculares propuestos en el primer nivel en las carreras de las Facultades de Arquitectura y Urbanismo; Ingeniería en Geología, Minas, Petróleos y Ambiental; y, Jurisprudencia, Ciencias Políticas y Sociales de la UCE.

Los resultados por Facultades no guardan similitudes, por tanto, los análisis deben realizarse por separado.

Se encuentra mejor relación en los resultados de FAU y FIGEMPA.

En las Facultades de Jurisprudencia y de Arquitectura hay una mejora del 2012 al 2014 y de éste período al 2016.

En Jurisprudencia se debe reforzar: el desarrollo del pensamiento y el razonamiento lógico, la inmersión en el mundo universitario, finalmente el reforzar el uso de herramientas tecnológicas. En el caso de Arquitectura: desarrollo de habilidades orales y escritas; deben realizar actividades que desarrollen el razonamiento verbal. 
En la FIGEMPA entre 2012 y 2014 existen 3 respuestas que manifiestan mejoras y 10 desmejoran los resultados de nivelación. En el análisis de 2012 a 2016 hay 3 que mejoran, 4 se mantienen igual y 6 empeoran. En el intervalo de 2014 a 2016 existen 9 mejoras y 4 retrocesos. Es visible un fuerte deterioro con la propuesta de la SENESCYT (2014) y una mejora significativa con la propuesta de la UCE; por tanto no existen elementos que permitan dar ilación a las tareas. Los aspectos a considerar para nuevos procesos pasan por reforzar: el desarrollo del pensamiento y el razonamiento lógico, la inmersión en el mundo universitario, finalmente el reforzar el uso de herramientas tecnológicas.

Los niveles de adaptabilidad en cuanto a sentirse identificado con los principios de interculturalidad como medio de convivencia socio-afectiva; así como los factores con los que se sienten identificados los estudiantes dan cuenta de un buen nivel de adaptabilidad, lo que conlleva a afirmar que hay adaptación a la Universidad, a su medio y condiciones. En el proceso de 2016 se llevó a través de la asignatura de Ética y Pensamiento Universitario (EPU) por medio de un curso virtual, aquello lleva a concluir que la propuesta pomposa de la Secretaría dilapida el tiempo es su propuesta, basta con un currículo adecuado y su conducción adecuada a través de la tecnología.

Se recomienda a futuro elaborar una nueva propuesta que, recogiendo las experiencias obtenidas en la presente investigación, garantice la inserción de los estudiantes en educación superior, con los conocimientos, características y destrezas requeridas para cada carrera y los principios del buen vivir aceptados.

Es pertinente para la Universidad investigar los efectos que se han producido por el cambio de docentes en este período. De la misma manera si los enfoques curriculares aportan a lograr mejores niveles de adaptabilidad de los bachilleres que ingresan a la Universidad.

\section{Bibliografía}

Asamblea Constituyente. (2008). Constitución de la República del Ecuador. Montecristi: Registro Oficial.

Asamblea Nacional. (2010). Ley Orgánica de Educación Superior. Quito: Registro Oficial.

Asamblea Nacional. (2012). Marco Legal educativo. Quito: Editogran.

Barreto, M., Lescano, D., Hidalgo, B., Inca, A., \& Cisneros, A. (12 de Septiembre de 2017). Sistema Nacional de Nivelación y Admisión. Obtenido de Universidad y Buen Vivir: http://www.snna.gob.ec/wp-content/themes/institucion/biblioteca.php

Carrera, C. (2017). Estudio comparativo de la eficiencia académica entre el curso propedeútico anterior al 2012-B y el curso de nivelación del sistema nacional de nivelación y admisión SNNA, en la Escuela Politécnica Nacional en el período 2011-2014. Quito.

Dirección General Académica. (2016). Informe SNNA. Universidad Central del Ecuador, DGAUCE, Quito. 
Maldonado, B. (2015). El Sistema Nacional de Admisión y Nivelación del Ecuador desde la perspectiva de la Universidad de las Fuerzas Armadas. San Gregorio, 43-60.

Ministerio de Educación. (2010). Nuevo Bachillerato Ecuatoriano. Quito.

Ministerio de Educación y Cultura. (1994). Breve Evolución Histórica del Sistema Educativo. En C. Poveda, G. Díaz, A. Abendaño, A. Benalcázar, S. Araujo, \& R. Arboleda, Informe OEIMinisterio 1994 (págs. 31-64). Quito: OEI.

Ocegueda, J., Miramontes, M., Moctezuma, P., \& Mungaray, A. (2017). Análisis comparado de la cobertura de la educación superior en Corea del Sur y Chile: una reflexión para México. (U. A. México, Ed.) Perfiles Educativos, XXXIX(155), 141-159.

Ortiz, J. (2010). Informes de nivelación FIGEMPA. Facultad de Ingeniería en Geología, Minas, Petróleos y Ambiental, Instituto de Ciencias Básicas, Quito.

Ramírez, R. (2013). Tercera Ola De Transformación De La Educación Superior En Ecuador. Hacia la constitucionalización de la sociedad del buen vivir. Quito: Secretaría Nacional de Educación Superior, Ciencia, Tecnología e Innovación.

SENESCYT. (2012). Sistema Nacional De Nivelación Y Admisión. Quito.

SENESCYT. (2014). Microcurrículos por áreas de conocimiento. Quito: Senescyt.

SENESCYT. (2014). Reglamento del Sistema Nacional de Nivelación y Admisión. Quito: Registro Oficial.

Senplades. (2010). Seminario Internacional de Admisión y Nivelación a la Universidad en América Latina: Diagnóstico y perspectivas. Quito: Hojas y signos.

Viera, P. (2017). Los que "triunfan" y los que "fracasan" (Des)igualdades sociales, logros educativos y emociones: el ingreso a la universidad pública en el Ecuador de las oportunidades. Conferencia Nacional "Educación Superior en el Ecuador. Investigaciones para una Nueva Agenda de Cambios Necesarios” (págs. 1-29). Quito: UASB-Digital.

Yamada, g., Castro, J., Bacigalupo, J., \& Velarde, L. (2013). Mayor acceso con menor calidad en la educación superior: algunasevidencias desde las habilidades de los estudiantes. Centro de Investigaciones de la Universidad del Pacífico, XL(72), 7-32. 